\title{
LYAPUNOV THEOREMS FOR BANACH SPACES
}

\author{
Y. LATUSHKIN AND S. MONTGOMERY-SMITH
}

\begin{abstract}
We present a spectral mapping theorem for semigroups on any Banach space $E$. From this, we obtain a characterization of exponential dichotomy for nonautonomous differential equations for $E$-valued functions. This characterization is given in terms of the spectrum of the generator of the semigroup of evolutionary operators.
\end{abstract}

\section{INTRODUCTION}

Let us consider an autonomous differential equation $y^{\prime}=A y$ in a Banach space $E$, where $A$ is a generator of continuous semigroup $\left\{e^{t A}\right\}_{t \geq 0}$; that is, the solution of the differential equation satisfies $y(t)=e^{t A} y(0), t \geq 0$. A classical result of A. M. Lyapunov (see, e.g., [DK]) shows that for bounded $A$, the spectrum $\sigma(A)$ of $A$ is responsible for the asymptotic behavior of $y(t)$. For example, if $\operatorname{Re} \sigma(A)<0$, then the trivial solution is uniformly asymptotically stable, that is, $\left\|e^{t A}\right\| \rightarrow 0$ as $t \rightarrow \infty$. This fact follows from the spectral mapping theorem (see, e.g., [N, p. 82]):

$$
\sigma\left(e^{t A}\right) \backslash\{0\}=\exp (t \sigma(A)), \quad t \neq 0,
$$

which always holds for bounded $A$.

For unbounded $A$, equation (1) is not always true. Moreover, there are examples of generators $A$ (see [N, p. 61]) such that even $\operatorname{Re}(\sigma(A)) \leq s_{0}<0$ does not guarantee $\sigma\left(e^{A}\right) \subset \mathbb{D}=\{|z|<1\}$ or $\left\|e^{t A}\right\| \rightarrow 0$ as $t \rightarrow \infty$. Since $\sigma(A)$ does not characterize the asymptotic behavior of the solutions $y(t)$, we would like to find some other characterization that still does not involve solving the differential equation (i.e., finding $\sigma\left(e^{t A}\right)$ ) .

In this article we solve precisely this problem in the following manner. Consider the space $L_{p}(\mathbb{R} ; E)$ of $E$-valued functions for $1 \leq p<\infty$ and the semigroup $\left\{e^{t B}\right\}_{t \geq 0}$ of evolutionary operations (also called weighted translation operators)

$$
\left(e^{t B} f\right)(x)=e^{t A} f(x-t), \quad t \geq 0,
$$

generated by the operator $B=-\frac{d}{d x}+A, x \in \mathbb{R}$. It turns out that it is $\sigma(B)$ in $L_{p}(\mathbb{R} ; E)$ that is responsible for the asymptotic behavior of $y(t)$ in $E$. For example, $\operatorname{Re}(\sigma(B))<0$ on $L_{p}(\mathbb{R} ; E)$ implies that $\left\|e^{t A}\right\| \rightarrow 0$ as $t \rightarrow \infty$ on $E$.

Received by the editors February 19, 1993.

1991 Mathematics Subject Classification. Primary 4706, 47B38; Secondary 34D20, 34G10.

Key words and phrases. Hyperbolicity, evolution family, exponential dichotomy, weighted composition operators, spectral mapping theorem. 
We will also consider the well-posed nonautonomous equation $y^{\prime}=A(t) y(t)$. Instead of the semigroup given by $(2)$, we consider in $L_{p}(\mathbb{R} ; E)$ the semigroup

$$
\left(e^{t D} f\right)(x)=U(x, x-t) f(x-t), \quad x \in \mathbb{R}, t \geq 0 .
$$

Here $U(t, s), t \geq s$, is the evolutionary family (propagator) for the nonautonomous equation. We will show that $\sigma(D)$ characterizes the asymptotic behavior of $y(t)$.

The order of proofs will be as follows. We will first show the spectral mapping theorem for the autonomous case (2). We will also consider a similar theorem for the evolutionary semigroup in the space of the periodic functions. This theorem will give us a variant of Greiner's spectral mapping theorem (see [N, p. 94]) for any $C_{0}$-semigroup $\left\{e^{t A}\right\}$ in a Banach space. This variant also is a direct generalization of Gerhard's spectral mapping theorem in Hilbert space for generators with resolvent bounded along $i \mathbb{R}$ (see [N, p. 95]). Then we will obtain the spectral mapping theorem for the nonautonomous case (3) using a simple change of variables argument to reduce it to the autonomous case (2).

We will be considering not only stability but also the exponential dichotomy (hyperbolicity) for the solutions of the equation $y^{\prime}=A(t) y(t)$ in $E$. In the theory of differential equations with bounded coefficients, exponential dichotomy is an important tool used, for example, in proving instability theorems for nonlinear equations and determining existence and uniqueness of bounded solutions and Green's functions (see, e.g., [DK]). The spectral mapping theorem given here for the semigroup (3) allows one to extend these ideas to the case of unbounded coefficients.

In turns out that the condition $0 \notin \sigma(D)$-or equivalently $\sigma\left(e^{t D}\right) \cap \mathbb{T}=\varnothing$, $t>0, \mathbb{T}=\{|z|=1\}$ on $L_{p}(\mathbb{R} ; E)$-is equivalent to the hyperbolic behavior of a special kind for the solutions. We will call this spectral hyperbolicity. Note that if the $U(t, s)$ are invertible for $(t, s) \in \mathbb{R}^{2}$, that is, (3) is extendible to a group, then the spectral hyperbolicity is the same as the exponential dichotomy (or hyperbolicity) in the usual (see, e.g., [DK]) sense. Therefore, the spectrum $\sigma\left(e^{t D}\right)$ for nonperiodic $A(\cdot)$ plays the same role in the description of exponential dichotomy as the spectrum of the monodromy operator does in the usual Floquet theory for the periodic case. However, ordinary hyperbolicity is not equivalent (see $[R])$ to spectral hyperbolicity in the semigroup case and thus cannot be characterized in terms of $\sigma\left(e^{t D}\right)$ only. For a Hilbert space, we were able to characterize hyperbolicity in terms of other spectral properties of $e^{t D}$.

Finally, the results of this article can be generalized to the case of the variational equation $y^{\prime}(t)=A\left(\varphi^{t} x\right) y(t)$ for a flow $\left\{\varphi^{t}\right\}$ on a compact metric space $X$ or for a linear skew-product flow $\hat{\varphi}^{t}: X \times E \rightarrow X \times E:(x, y) \mapsto$ $\left(\varphi^{t} x, \Phi(x, t) y\right), t \geq 0$ (see [CS, H, LS, SS] and references contained therein). Here $\Phi: X \times \mathbb{R}_{+} \rightarrow L(E)$ is a cocycle over $\varphi^{t}$, that is, $\Phi(x, t+s)=$ $\Phi\left(\varphi^{t} x, s\right) \Phi(x, t), \Phi(x, 0)=I$. Let us recall (see [SS]) that one of the purposes of the theory of linear skew-product flows was to aid in studying the equation $y^{\prime}=A(t) y$ for the case of almost periodic $A(\cdot)$. To answer the question when $\hat{\varphi}^{t}$ is hyperbolic (or Anosov), instead of (3) one considers the semigroup of so-called weighted composition operators (see $[\mathrm{CS}, \mathrm{J}, \mathrm{LS}]$ ) on $L_{p}(X ; \mu ; E)$ :

$$
\left(T^{t} f\right)(x)=\left(\frac{d \mu \circ \varphi^{-t}}{d \mu}\right)^{1 / p} \Phi\left(\varphi^{-t} x, t\right) f\left(\varphi^{-t} x\right), \quad x \in X, t \geq 0
$$


Here $\mu$ is a $\varphi^{t}$-quasi-invariant Borel measure on $X$. As above, the condition $\sigma\left(T^{t}\right) \cap \mathbb{T}=\varnothing$ is equivalent to the spectral hyperbolicity of the linear skew-product flow $\hat{\varphi}^{t}$. The spectral hyperbolicity coincides with the usual hyperbolicity if $\Phi(x, t), x \in X, t \geq 0$, are invertible or compact operators. Unlike the finite-dimensional case (see [M]), the hyperbolicity of $\hat{\varphi}^{t}$ does not generally imply the condition $\sigma\left(T^{t}\right) \cap \mathbb{T}=\varnothing$. For Hilbert space the condition of hyperbolicity of $\hat{\varphi}^{t}$ can also be described in terms of other spectral properties of $T^{t}$. We will not include these generalizations in this paper.

We point out that the investigation of evolutionary operators (2) and (3) has a long history [Ho]. More recently, significant progress has been made in [BG, N, P, R] (see [R] for detailed bibliography), and this list does not pretend to be complete. A detailed investigation of weighted composition operators (4) for Hilbert spaces $E$ and connections with the spectral theory of linear skew-product flows [SS] and other questions of dynamical systems theory and a bibliography may be found in [LS].

Notation. $L(E)$ (correspondingly $L_{s}(E)$ ) denotes the set of bounded operators on $E$ with the uniform (correspondingly strong) topology; $\rho(A)$ denotes the resolvent set of the operator $A ; \mid$ denotes the restriction of an operator; $C_{b}(\mathbb{R} ; E)$ denotes the space of continuous bounded $E$-valued functions on $\mathbb{R}$ with the supremum norm, and $C_{b}^{0}(\mathbb{R} ; E)$ denotes the subspace of functions vanishing at infinity.

\section{Autonomous case}

Let $A$ be a generator of any $C_{0}$-semigroup in a Banach space $E$. Consider the $C_{0}$-semigroup (2) on the space $L_{p}([0,2 \pi) ; E), 1 \leq p<\infty$, that is, $\left(e^{t B} f\right)(x)=e^{t A} f((x-t)(\bmod 2 \pi))$.

Theorem 1. The following are equivalent:

(1) $1 \in \rho\left(e^{2 \pi A}\right)$ on $E$;

(2) $0 \in \rho(B)$ on $L_{p}([0,2 \pi) ; E)$;

(3) $1 \in \rho\left(e^{2 \pi B}\right)$ on $L_{p}([0,2 \pi) ; E)$.

In the main part of the proof $(2) \Rightarrow(1)$, we modify the idea of [CS]. Let us assume that (2) is fulfilled, but for each $0<\varepsilon<\frac{1}{2}$, there is a $y \in E$ such that $\left\|e^{2 \pi A} y-y\right\|<\varepsilon$ and $\|y\|=1$ (and hence $\left\|e^{2 \pi A} y\right\| \geq \frac{1}{2}$ ). Let $\rho(x)=\frac{3}{2 \pi} x-1$ for $x \in\left[\frac{2 \pi}{3}, \frac{4 \pi}{3}\right), \rho(x)=0$ for $x \in\left[0, \frac{2 \pi}{3}\right)$, and $\rho(x)=$ 1 for $x \in\left[\frac{4 \pi}{3}, 2 \pi\right)$. Define the function $f \in L_{p}([0,2 \pi) ; E)$ by $f(x)=$ $(1-\rho(x)) e^{(2 \pi+x) A} y+\rho(x) e^{x A} y, x \in[0,2 \pi)$. Then for $c=\max \left\{\left\|e^{x A}\right\|: x \in\right.$ $[0,2 \pi)\}$, one has $\left\|e^{2 \pi A} y\right\|=\left\|e^{(2 \pi-x) A} e^{x A} y\right\| \leq c\left\|e^{x A} y\right\|$. But, in contradiction with (2),

$$
\|f\|_{p}^{p} \geq \int_{4 \pi / 3}^{2 \pi}\left\|e^{x A} y\right\|^{p} d x \geq \frac{2 \pi}{3} c^{-p}\left\|e^{2 \pi A} y\right\|^{p} \geq \frac{2 \pi}{3} c^{-p} 2^{-p}
$$

and

$$
\|B f\|_{p} \leq \frac{2 \pi}{3} c \varepsilon
$$

Theorem 1 implies the following variant of Greiner's spectral mapping theorem (see [N, p. 94]) for a $C_{0}$-semigroup $\left\{e^{t A}\right\}$ in Banach space $E$. 
Theorem 2. $1 \in \rho\left(e^{2 \pi A}\right)$ if and only if (a) $i \mathbb{Z} \subset \rho(A)$ and (b) there is a constant $C$ such that for any finite sequence $\left\{y_{k}\right\} \subset E$

$$
\left\|\sum_{k}(A-i k)^{-1} y_{k} e^{-i k x}\right\|_{L_{p}([0,2 \pi) ; E)} \leq C\left\|\sum_{k} y_{k} e^{-i k x}\right\|_{L_{p}([0,2 \pi) ; E)} .
$$

Obviously, if $E$ is a Hilbert space and $p=2$, then Parseval's identity allows one to replace (b) by the condition $\sup \left\{\left\|(A-i k)^{-1}\right\|: k \in \mathbb{Z}\right\}<\infty$. This gives the famous spectral mapping theorem of Gerhard [N, p. 95].

Let us consider (2) on $L_{p}(\mathbb{R} ; E), 1 \leq p<\infty$. The spectrum $\sigma(B)$ now is invariant under the translations along the imaginary axis. Moreover, we have the following result.

Theorem 3. For each $t>0$ the following are equivalent:

(1) $\sigma\left(e^{t A}\right) \cap \mathbb{T}=\varnothing$ on $E$;

(2) $0 \in \rho(B)$ on $L_{p}(\mathbb{R} ; E)$;

(3) $\sigma\left(e^{t B}\right) \cap \mathbb{T}=\varnothing$ on $L_{p}(\mathbb{R} ; E)$.

Thus, the spectral mapping theorem is valid for (2). If $\left\{e^{t A}\right\}_{t \in \mathbb{R}}$ is a group, then (1) is the same as the exponential dichotomy of the autonomous equation $y^{\prime}=A y$ on $\mathbb{R}$.

\section{NonAUtONOMOUS CASE}

Consider the well-posed nonautonomous equation $y^{\prime}(t)=A(t) y(t)$. By "well-posed" we mean that we assume the existence of a jointly strongly continuous evolutionary family $U(t, s) \in L_{s}(E), t \geq s$, with the properties $U(t, t)=$ $I, U(t, r)=U(t, s) U(s, r)$, and $\|U(r, s)\| \leq C e^{\beta(t-s)}, t \geq r \geq s$. In fact, $U$ is a propagator for the equation $y^{\prime}(t)=A(t) y(t)$, that is, $y(t)=U(t, s) y(s)$. The spectral mapping theorem is valid for $(3)$.

Theorem 4. Let (3) be a $C_{0}$-semigroup on $L_{p}(\mathbb{R} ; E), 1 \leq p<\infty$. Then $\sigma(D)$ is invariant under translations along the imaginary axis and the following are equivalent:

(1) $0 \in \rho(D)$ on $L_{p}(\mathbb{R} ; E)$;

(2) $\sigma\left(e^{t D}\right) \cap \mathbb{T}=\varnothing$ on $L_{p}(\mathbb{R} ; E), t>0$.

To outline the proof of $(1) \Rightarrow(2)$, let us consider the semigroup $\left(e^{t B} h\right)(s, x)=$ $U(x, x-t) h(s-t, x-t),(s, x) \in \mathbb{R}^{2}, t>0$, on the space $L_{p}(\mathbb{R} \times \mathbb{R} ; E)=$ $L_{p}\left(\mathbb{R} ; L_{p}(\mathbb{R} ; E)\right)$ and perform a change of variables $u=s+x, v=x$. More precisely, consider the isometry $J$ on $L_{p}(\mathbb{R} \times \mathbb{R} ; E)$ defined by $(J h)(s, x)=$ $h(s+x, x)$. Then one has that $J e^{t B}=\left(I \otimes e^{t D}\right) J$ and $J B=(I \otimes D) J$, where $A_{1} \otimes A_{2}$ means that $A_{1}$ acts on $h(\cdot, x)$ and $A_{2}$ acts on $h(s, \cdot)$. Since $e^{t B}$ can be written as $\left(e^{t B} f\right)(s)=e^{t D} f(s-t)$ for $f: \mathbb{R} \rightarrow L_{p}(\mathbb{R} ; E): s \mapsto h(s, \cdot)$, one can apply to $e^{t B}$ the part (2) $\Rightarrow(3)$ of Theorem 3 .

Definition. The evolutionary family $\{U(x, s)\}_{x \geq s}$ is called hyperbolic if there exists a projection-valued function $P: \mathbb{R} \rightarrow L(E), P \in C_{b}\left(\mathbb{R} ; L_{s}(E)\right)$, and $M$, $\lambda>0$ such that for all $x \geq s$ :

(1) $P(x) U(x, s)=U(x, s) P(s)$; and

(2) $\|U(x, s) y\| \leq M e^{-\lambda(x-s)}\|y\|$ if $y \in \operatorname{Im} P(s)$; $\|U(x, s) y\| \geq M^{-1} e^{\lambda(x-s)}\|y\|$ if $y \in \operatorname{Ker} P(s)$. 
The evolutionary family $\{U(x, s)\}_{x \geq s}$ is called spectrally hyperbolic if, in addition:

(3) $\operatorname{Im} U(x, s) \mid \operatorname{Ker} P(s)$ is dense in $\operatorname{Ker} P(x)$.

Note that the second inequality in (2) implies only left invertibility of the restriction $U(x, s) \mid \operatorname{Ker} P(s)$, while (3) guarantees its invertibility. If the evolutionary family $\{U(x, s)\}_{(x, s) \in \mathbb{R}^{2}}$ consists of invertible operators, then spectral hyperbolicity is equivalent to the hyperbolicity and is the same as exponential dichotomy [DK] of the equation $y^{\prime}=A(t) y$ on $\mathbb{R}$. If $\operatorname{dim} \operatorname{Ker} P(s)<d<\infty$, then obviously (2) always implies (3). This also happens if the $U(x, s)$ are compact operators in $E$ [R1].

Theorem 5. The evolutionary family $\{U(x, s)\}$ on the separable Banach space $E$ is spectrally hyperbolic if and only if $0 \in \rho(D)$ on $L_{p}(\mathbb{R} ; E)$.

Remark. The space $L_{p}$ may be replaced by the space $C([0,2 \pi) ; E)$ in Theorem 1 and by $C_{b}^{0}(\mathbb{R} ; E)$ in Theorems 2 to 5 . The separability assumption in Theorem 5 was recently removed [LR].

A remarkable observation in [R] shows that the hyperbolicity of $\{U(x, s)\}_{x \geq s}$ (unlike the spectral hyperbolicity) does not generally imply (2) in Theorem 4 for infinite-dimensional $E$. However, we are able to give the following characterization of hyperbolicity under the assumptions that $E$ is a Hilbert space and that for some $r>0$ the function $x \mapsto U(x+r, x)$ is a continuous function from $\mathbb{R}$ to $L(E)$.

Theorem 6. The evolutionary family $\{U(x, s)\}_{x \geq s}$ is hyperbolic on a separable Hilbert space $E$ if and only if there exists a projection $\mathscr{P}$ on $L_{2}(\mathbb{R} ; E)$ such that for some $t>0$ :

(1) $e^{t D \mathscr{P}}=\mathscr{P} e^{t D}$

(2) $\sigma\left(e^{t D} \mid \operatorname{Im} \mathscr{P}\right) \subset \mathbb{D}$;

(3) $e^{t D} \mid \operatorname{Ker} \mathscr{P}$ is left invertible and $\sigma\left(\left(e^{t D} \mid \operatorname{Ker} \mathscr{P}\right)^{\dagger}\right) \subset \mathbb{D}$;

(4) $\operatorname{Ker} \mathscr{P} \ominus \bigcap_{n \geq 0} \operatorname{Im}\left(e^{n t D} \mid \operatorname{Ker} \mathscr{P}\right)$ is invariant with respect to multiplications by the functions from $C_{b}(\mathbb{R} ; \mathbb{R})$.

Each projection $\mathscr{P}$ with these properties has a form $(\mathscr{P} f)(x)=P(x) f(x)$ for a projection-valued function $P \in C_{b}(\mathbb{R} ; L(E))$.

For the left-invertible operator $T$ the notation $T^{\dagger}$ stands for its MoorePenrose left inverse: $T^{\dagger} u=v$ if $u=T v$, and $T^{\dagger} u=0$ for $u \perp \operatorname{Im} T$. Note that (1), (2), (3) imply the left invertibility of $z I-e^{t D}$ for all $z \in \mathbb{T}$ and the formula $\mathscr{P}=\frac{1}{(2 \pi i)} \int_{\mathbb{T}}\left(z I-e^{t D}\right)^{\dagger} d z$ which gives the Riesz projection on $\sigma\left(e^{t D}\right) \cap \mathbb{D}$ if $\sigma\left(e^{t D}\right) \cap \mathbb{T}=\varnothing$.

\section{REFERENCES}

[BG] A. Ben-Artzi and I. Gohberg, Dichotomy of systems and invertibility of linear ordinary differential operators, Oper. Theory Adv. Appl., vol. 56, Birkhaüser, Basel, 1992, pp. 90-119.

[CS] C. Chicone and R. Swanson, Spectral theory of linearizations of dynamical systems, J. Differential Equations 40 (1981), 155-167.

[DK] J. Daleckij and M. Krein, Stability of differential equations in Banach space, Transl. Math. Mono., vol. 43, Amer. Math. Soc., Providence, RI, 1974.

[H] J. Hale, Asymptotic behavior of dissipative systems, Math. Surveys Monographs, vol. 25, Amer. Math. Soc., Providence, RI, 1988. 
[Ho] J. S. Howland, Stationary scattering theory for time-dependent hamiltonians, Math. Annal. 207 (1974), 315-335.

[J] R. Johnson, Analyticity of spectral subbundles, J. Differential Equations 35 (1980), 366-387.

[LM] Y. Latushkin and S. Montogomery-Smith, Evolutionary semigroups and Lyapunov theorems in Banach spaces, J. Funct. Anal. (to appear).

[LR] Y. Latushkin and T. Randolph, Dichotomy of differential equations on Banach spaces and an algebra of weighted translation operators, Trans. Amer. Math. Soc., submitted.

[LS] Y. Latushkin and A. Stepin, Weighted translations operators and linear extensions of dynamical systems, Russian Math. Surveys 46 (1991), 95-165.

[N] R. Nagel (ed.), One parameters semigroups of positive operators, Lecture Notes in Math., vol. 1184, Springer-Verlag, Berlin, 1984.

[M] J. Mather, Characterization of Anosov diffeomorphisms, Indag. Math. 30 (1968), 479-483.

[P] K. Palmer, Exponential dichotomy and Fredholm operators, Proc. Amer. Math. Soc. 104 (1988), 149-156.

[R] R. Rau, Hyperbolic evolution groups and exponentially dichotomic evolution families, J. Funct. Anal. (to appear).

[R1] Hyperbolic evolutionary semigroups on vector-valued function spaces, Semigroup Forum 48 (1994), 107-118.

[SS] R. Sacker and G. Sell, Dichotomies for linear evolutionary equations in Banach spaces, IMA preprint no. 838, 1991.

Department of Mathematics, University of Missouri, Columbia, Missouri 65211

E-mail address, Y. LATUSHKIN: mathyl@mizzou1.missouri.edu

E-mail address, S. MONTGOMERY-SMITH: stephen@mont.cs.missouri.edu 